\title{
High Performance Simulations to Support Real-time COVID19 Response
}

\author{
Madhav Marathe \\ Network Systems Science and Advanced Computing Division \\ Biocomplexity Institute \& Dept. of Computer Science \\ University of Virginia \\ marathe@virginia.edu
}

\begin{abstract}
The COVID-19 pandemic represents an unprecedented global crisis and serves as a reminder of the social, economic and health burden of infectious diseases. The ongoing trends towards urbanization, global travel, climate change and a generally older and immuno-compromised population continue to make epidemic planning and control challenging. Recent advances in computing, AI, and bigdata have created new opportunities for realizing the vision of real-time epidemic science.

In this talk I will describe our group's work developing scalable and pervasive computing-based concepts, theories and tools for planning, forecasting and response in the event of epidemics. I will draw on our work in supporting federal agencies as they plan and respond to the COVID-19 pandemic outbreak. I will end the talk by outlining directions for future work.
\end{abstract}

\section{CCS Concepts/ACM Classifiers}

Computing methodologies $\rightarrow$ Modeling and simulation

\section{Author Keywords}

pandemic; computational epidemiology; big data; AI; high performance computing

\section{BIOGRAPHY}

Madhav Marathe is the division director of the Network Systems Science and Advanced Computing Division at the Biocomplexity Institute and Initiative, and a Distinguished Professor at the University of Virginia. His research interests are in network science, foundations of computing, computational epidemiology, and high-performance computing. Before joining UVA, he held positions at Virginia Tech and Los Alamos National Laboratory. He is a Fellow of the IEEE, ACM, SIAM, and AAAS.

Permission to make digital or hard copies of part or all of this work for personal or classroom use is granted without fee provided that copies are not made or distributed for profit or commercial advantage and that copies bear this notice and the full citation on the first page. Copyrights for third-party components of this work must be honored. For all other uses, contact the Owner/Author.

SIGSIM-PADS '20, 7une 15-17, 2020, Miami, FL, USA

(C) 2020 Copyright is held by the owner/author(s).

ACM ISBN 978-1-4503-7592-4/20/06.

https://doi.org/10.1145/3384441.3395993

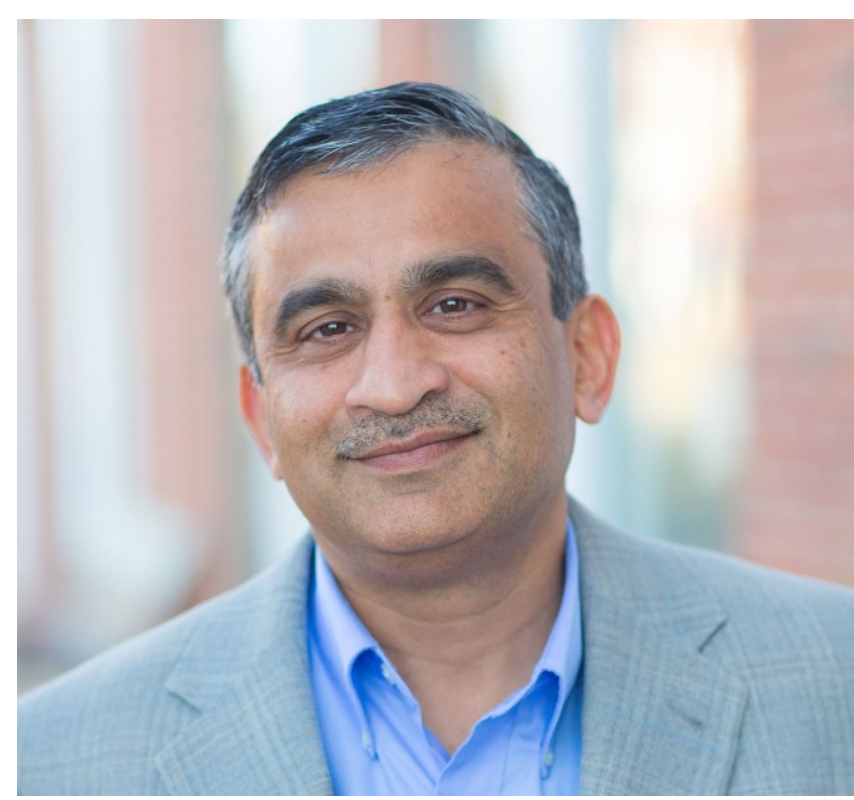

\section{REFERENCES}

[1] Eubank S, Guclu H, Kumar A, Marathe M, Srinivasan A, Toroczkai Z, Wang N (2004). Modelling disease outbreaks in realistic urban social networks. Nature, 429(6988): 180-184.

[2] Marathe M, Vullikanti A (2013) Computational Epidemiology. Communications of the ACM (CACM), 56(7):88-96.

[3] https://covid19.biocomplexity.virginia.edu/

\section{ACKNOWLEDGEMENTS}

This work was partially supported by National Institutes of Health (NIH) Grant 1R01GM109718, NSF BIG DATA Grant IIS1633028, NSF DIBBS Grant ACI1443054, NSF Grant No.: OAC1916805, NSF Expeditions in Computing Grant CCF-1918656 CCF-1917819, US Centers for Disease Control and Prevention 75D30119C05935, DTRA subcontract/ARA S-D00189-15-TO-01UVA, and a collaborative seed grant from the UVA Global Infectious Disease Institute. 